\title{
N-back tasks for spatial and verbal working memory using Near- Infrared Spectroscopy
}

\author{
Kota KANO*, Hisaya TANAKA* \\ * Kogakuin University, 2665-1 Nakano-cho, Hachioji-shi, Tokyo, 193-0015 Japan \\ em19003@ns.kogakuin.ac.jp hisaya@cc.kogakuinac.ac.jp
}

\begin{abstract}
The purpose of this study is to create new working-memory tests based on the N-back continuous performance task to account for verbal and visual acuity. We created two new N-back tasks: one for verbal working-memory (VWM) and another for spatial working memory (SWM). Like the conventional numeric working-memory (NWM) N-back test, these new types detect the same oxyhemoglobin reactions of the frontal lobe of the human brain. Thus, the responses of oxyhemoglobin during the various workingmemory tasks were measured using near-infrared spectroscopy. By evaluating NWM, VWM, and SWM, the number of trials in which activation was observed and the correct answer rates were compared. Results show that both VWM and SWM measured on par with NWM based on the Wilcoxon's rank-sum test. In the prefrontal cortex, there were no differences in oxyhemoglobin activity found to correspond to the different types of $\mathrm{N}$-tasks. This suggests that there may be a common domain for each type of cognitive function in the subjects.
\end{abstract}

Keywords: Near-Infrared Spectroscopy, working memory, N-back task

\section{Introduction}

In recent years, near-infrared spectroscopy (NIRS) has been used as a clinical tool in medicine and education, because it can detect and record human brain functions safely and easily [1]. The frontal lobe is responsible for the highest brain activities in humans, and it is home to a variety of diseases, such as dementia and mental illness. It is known that working memory, a frontal-lobe function, decreases with dementia (e.g., Alzheimer's) [2].

Consequently, many attempts have been made to quantify and evaluate frontal-lobe functions. In April 2009, the Ministry of Health, Labor and Welfare approved the use of the optical cognitive task of language fluency for cognitive tasks for differential diagnoses of depression symptoms using optical topography testing. NIRS can be used to harmlessly monitor changes in blood concentrations of oxyhemoglobin, deoxyhemoglobin, and total hemoglobin on the brain surface. It is suitable for measuring changes of blood flow in the frontal lobe and is perfect for assessing $\mathrm{N}$ back tasks.

Working memory is a biological system that temporarily stores and retrieves information necessary for complex active cognitive tasks, such as language understanding, learning, and inference. This memory type is capable of flexibly coping with a variety of realtime activities and tasks. Furthermore, it acts as a phonological loop related to verbal information processing and provides visuo-spatial processing to keep track of non-verbalizable information, including visual images. Working memory can thus be used to extract information stored in long-term memory and make it usable in addition to holding temporary information [2].

Working memory depends on the frontal-lobe association area, which distinguishes the types of information retained. These functions together comprise a domainspecific working-memory module for the human mind [3] [4].

The N-back test was developed to measure and assess working-memory functions. In a typical N-back test, onedigit numbers are presented randomly to measure a subject's working memory. Metrics are obtained by calculating the accurate recollection of a presented number from $\mathrm{N}$-times earlier. Conventional numerical working-memory (NWM) tasks are good for evaluations of numerical capability, but they do not adequately evaluate other types of operations, such as verbal and visual.

For this study, new verbal working-memory (VWM) and spatial working-memory (SWM) tasks were created to measure working memory using information other than numbers. The purpose of this study is, therefore, to compare the results of oxyhemoglobin measurement across all three types of $\mathrm{N}$-tasks.

\section{Experiment outline}

\section{$2.1 \quad$ Experimental purpose}

NIRS measurements were performed for NWM, VWM, and SWM testing. We examined the frontal-lobe activities of subjects and compared them across the three task-types.

\subsection{Measuring instrument}

An OEG-16 NIRS device manufactured by Spectratech was used. It comprised six light-sending 
probes and six light-receiving probes (Figure 1), leveraging near-infrared light of two wavelengths $(\sim 770$ and $880 \mathrm{~nm}$ ) to measure the concentrations of oxygenated (i.e., OxyHb) and deoxygenated hemoglobin (i.e., DeoxyHb) $2-3 \mathrm{~cm}$ below the scalp. the sum of the two is TotalHb. OEG-16 measures these hemoglobin changes using a total of 16 channels. The light-sending and the light-receiving probes were arranged at intervals of $3 \mathrm{~cm}$, and all channels fit within a $15 \times 3-\mathrm{cm}$ area. The datasampling interval was $0.655 \mathrm{~s}$. When mounting the OEG16 , the center of the holder upon whom probe was installed was positioned at the Fpz midline sagittal plane of the skull according to International 10-20 system (Figure 2) [4].

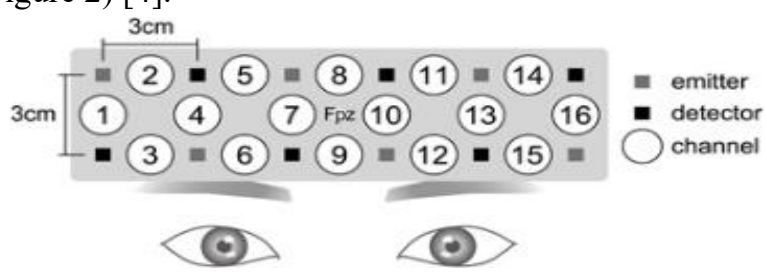

Figure 1: NIRS channel definition The $10-20$ system The $10 \%$ system
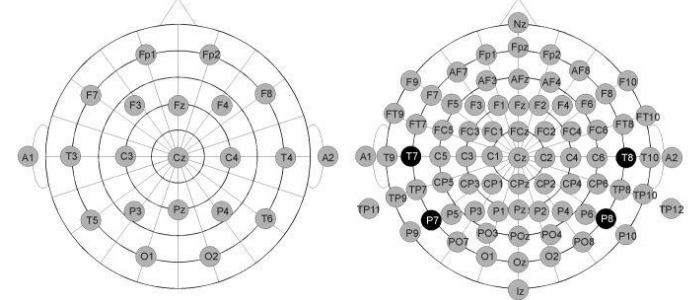

Figure 2: International The 10-20 system

\subsection{Experimental procedure}

The experiment was performed on five healthy subjects in their 20s. Figure 3 shows the procedure of this experiment. The distance between the participant's face and the monitor was set to $\sim 50 \mathrm{~cm}$. There were no moving objects and none having numbers or letters in the participant's field of view other than test objects, and the experimenter sat directly behind the participant. The task procedure was fully explained prior to the experiment. All subjects randomly performed four NWM trials, four SWM trials, and four VWM trials. Rest conditions were established beforehand for baseline measurement of blood flow. In the resting state, a white cross was visible on the screen.

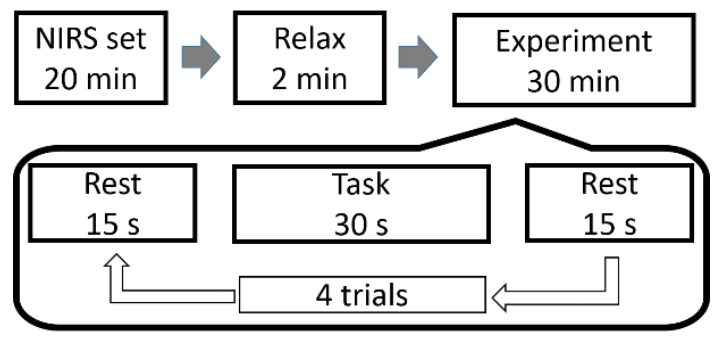

Figure 3: Experiment flow

\section{$2.4 \quad$ N-back test}

Nine stimuli corresponding to each task were displayed on the experimental screen. $\mathrm{N}$ was set to 3 , and the task was 3-back, meaning that a displayed stimulus is the same as that displayed three times earlier (Figure 4). The subject pressed a button as quickly as possible when the current screen matched the three previous stimuli. The number of correct answers was then counted. Each stimulus was displayed for $0.5 \mathrm{~s}$ with intervals of $1.5 \mathrm{~s}$. A total of 15 stimuli were presented for each trial, and four stimulus matches were included.

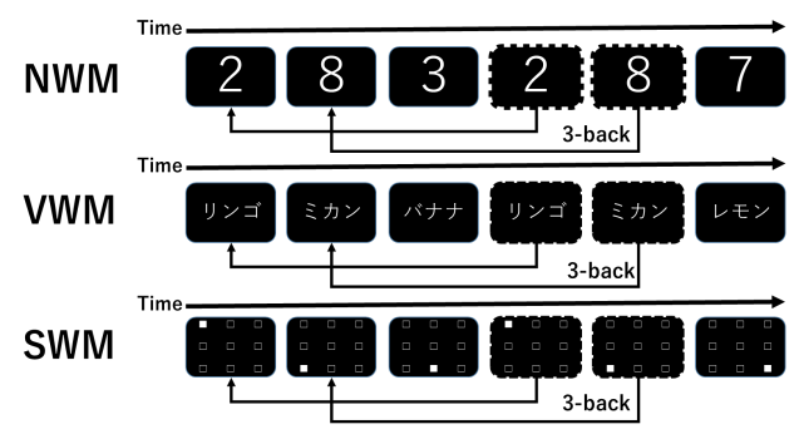

Figure 4: Working-memory task (3-back)

\subsection{NWM}

Single-digit numbers from 1-9 were displayed one at a time. The subject memorized the numbers and pressed the designated button when the number displayed matched that displayed three times earlier. Figure 5 shows an example of the stimulus used for the NWM) task.

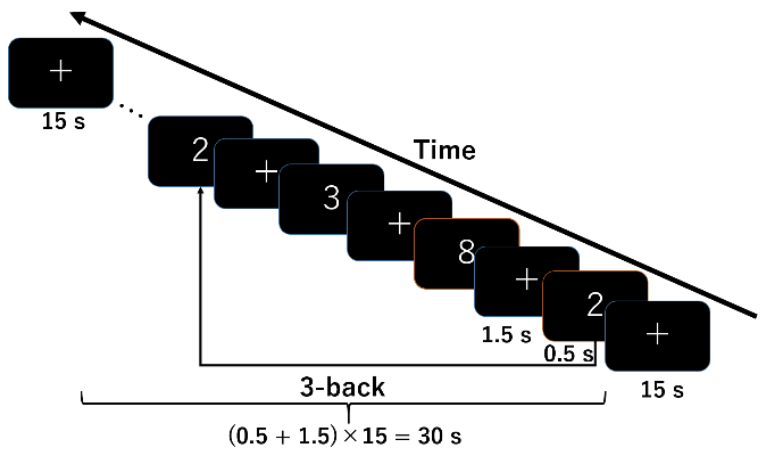

Figure 5: Presentation order of 3-back NWM tasks

\section{$2.6 \quad$ SWM}

Nine squares were displayed, and the subject remembered the position of the white square. They then pressed a designated button when the position of the white square was the same as the position of the one three times earlier. Figure 6 shows an example of the stimuli used SWM task.

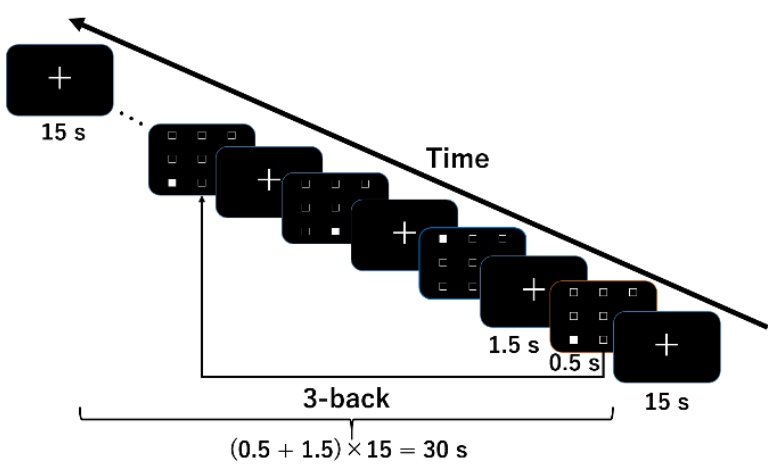

Figure 6: Presentation order of 3-back SWM tasks 
Table 1:Experimental activation channels

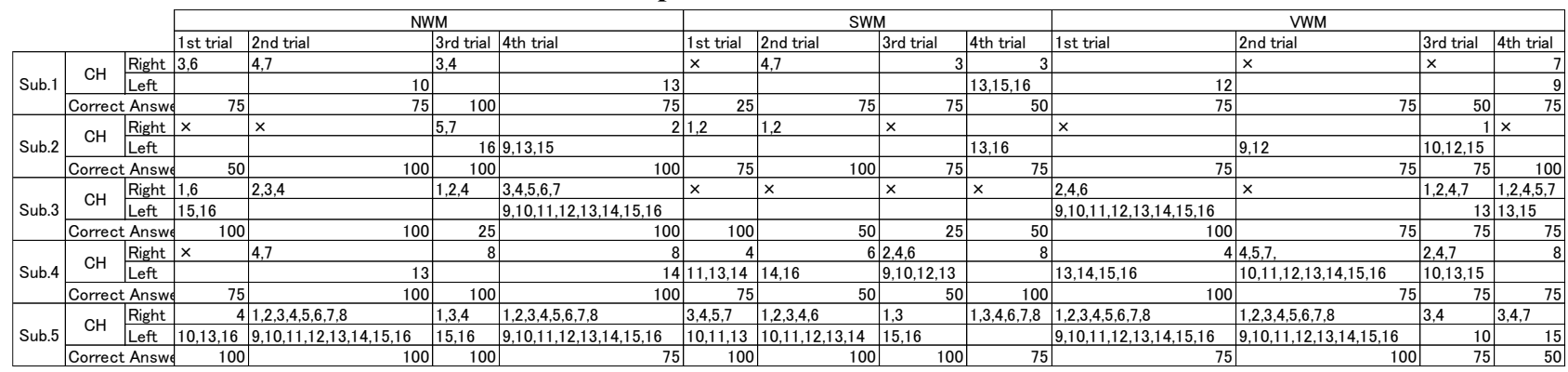

\section{$2.7 \quad$ VWM}

Nine fruit-related words were displayed on the screen for this task. The subject memorized the words, and, if the word displayed on the screen was the same as the one displayed three times earlier, he or she pressed the designated button. The words were unified into one category to integrate the stimuli. Figure 7 shows an example of the stimuli used in the experiment in the VWM task.

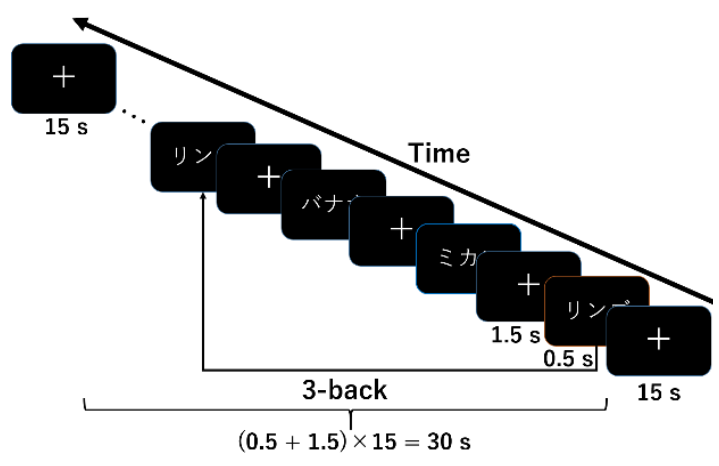

Figure 7: Presentation order of 3-back VWM tasks

\section{Results}

Of oxyHb, deoxyHb, and TotalHb, the change in oxyHb was the most sensitive to regional cerebral blood flow and blood-oxygen-level-dependent (BOLD) imaging signals. We used an oxyHb variation for analysis, because it had the highest correlation with BOLD signals [5][6]. The average value of oxyHb change in the rest section was also calculated. It was baseline corrected, and a 5-point moving average filter was applied.

Table 1 summarizes the activation channels and the correct answer rate for the first-to-fourth trials of NWM, SWM, and VWM for each of the five subjects. The activated channel was the one in which the amount of change in oxyhemoglobin increased in the frontal lobe during the task compared with the rest prior to the task. Specifically, the active channel was selected as one whose change in oxyhemoglobin showed a positive value $15 \mathrm{~s}$ after the start of the task ( $30 \mathrm{~s}$ after the start of the trial), which was the middle of the task section of NWM, SWM, and VWM. The right and left channels in Table 1, represented by rows associated with each subject, indicate that the channels were located in the right and left hemispheres, respectively. The correct answer rate for each trial was measured from the correct button pressed from the time of each stimulus (four matches) to the next, indicating the proportion of subjects who answered correctly during the four matches. Specifically, if the subject answered correctly four times out of the four matches, the correct answer rate would have been $100 \%$. NWM, SWM, and VWM were measured 60 times total, 20 times for each task, for five subjects. The number of trials in which frontal-lobe activity was observed during NWM, SWM, and VWM was 17, 14, and 15 , respectively.

Figure 8 shows the average correct answer rate (\%) for all trials. Activation of the frontal lobe during NWM, SWM, and VWM was indicated using trials that did not show activation. The average correct rate for all trials, that for trials having activation in the frontal lobe during NWM, SWM, and VWM, and those without activation were $77.46 \%, 81.12 \%$, and $66.07 \%$, respectively. The correct answer rate in the trials in which the frontal lobe was activated during the task and those in which the frontal lobe was not activated were tested using Wilcoxon's rank-sum test. As a result, a significant difference was observed between the two groups at a significance level of $5 \%$.

Figure 8 shows a graph obtained by averaging the changes in oxyhemoglobin during NWM, SWM, and VWM of the five subjects. For averaging, we used data from trials that were activated during the task.

Figure 9 shows the time change from the baseline oxyhemoglobin reflecting brain activity. The horizontal axis represents time (s), and the vertical axis represents the amount of change in oxyhemoglobin $(\mathrm{mmol} / \mathrm{L})$. The section surrounded by the dotted line in Figure 9 indicates the section in which the task was performed.

Figure 10 shows the results of integrating and comparing the changes in oxyhemoglobin in the NWM, SWM, and VWM task sections. The vertical axis represents the changes in oxyhemoglobin $\left(\mathrm{mmol} / \mathrm{L}^{2}\right)$.

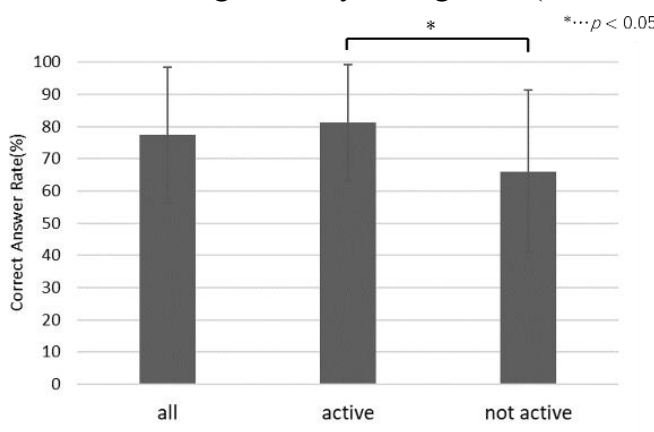

Figure 8: Comparison of activation correct answer rates (all, active, not active) 


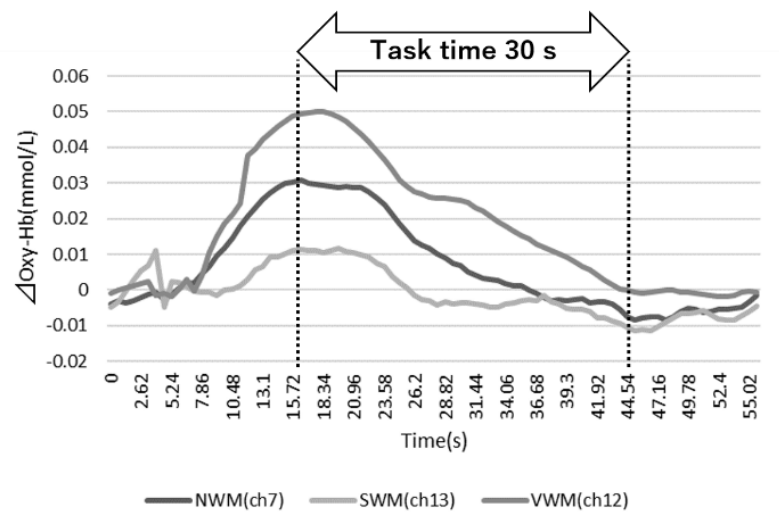

Figure 9: Changes in oxygenated hemoglobin during each task (3-back)

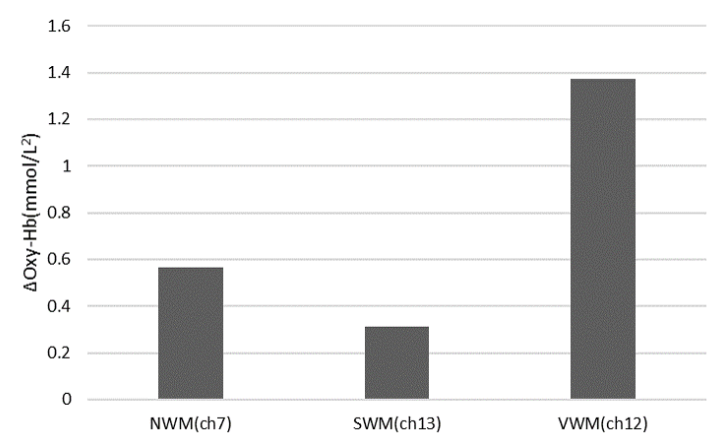

Figure 10: Change in oxygenated hemoglobin for each task(3-back)

\section{Discussion}

From Table 1, there was no significant difference between the number of trials in which activation was observed in the frontal lobe in the commonly used NWM and that with SWM and VWM. Figure 9 also shows that frontal-lobe activity during SWM and VWM increased in the task section, as it did during NWM. From this, it can be assumed that SWM and VWM can be used for working-memory tasks like NWM.

From Figure 8, it can be seen that the correct answer rate for trials not showing activation in the frontal lobe was lower than the correct answer rate for trials that showed activation in the frontal lobe during NWM, SWM, and VWM. This suggests that there was a positive correlation between frontal activation and workingmemory ability.

From the activation channels in Table 1, it can be seen that the channels activated by subjects during NWM were different. Similarly, it was found that activated channels differed between subjects during SWM and VWM. This suggests that there was no difference in the reaction site of the frontal lobe based on the type of information in working memory. On the other hand, the channels activated by the subjects were found to be common regardless of the type of task. This suggests that the reaction site of working memory in the prefrontal cortex was more affected by the subject's dominant hemisphere than by the type of information.

From Figure 9, it can be seen that the change in oxyhemoglobin during each task increased in the order of
VWM, NWM, and SWM. This suggests that working memory can be used more with language tasks than with numerical tasks.

\section{Conclusion}

In this study, we created NWM and SWM and compared it with VWM using NIRS. The frontal-lobe activity during each task was then compared. We found that SWM and VWM could be used as working-memory tasks, because there was no significant difference between the number of trials in which NWM activated the frontal lobe and that of SWM and VWM trials. Additionally, the correct answer rates tended to be higher depending on the presence or absence of activation in the frontal lobe $(\mathrm{p}<0.05)$. In the future, it will be necessary to examine the spatial domain of working memory by further increasing the numbers of subjects.

\section{References}

[1]. Baddeley, A. (1986). Working memory. Oxford: Oxford University Press.

[2]. Goldman-Rakic, P.S. (1994). The issue of memory in the study of prefrontal function. In A.-M. Thierry, J. Glowinski, P.S. Goldman-Rakic, \& Y. Christen (Eds.), Motor and cognitive functions of the prefrontal cortex (pp. 112-121). Berlin: SpringerVerlag.

[3]. Goldman-Rakic, P.S. (1996). The prefrontal landscape: Implications of functional architecture for understanding human mentation and the central executive. Philosophical Transactions of the Royal Society of London: B. 351, 1445-1453

[4]. Okamoto, M., Dan, H., Sakamoto, K., Takeo, K., Shimizu, K., Kohno, S., Dan, I. (2004). 3dimensional probabilistic anatomical craniocerebral correlation via the international 10-20 system oriented for transcranial functional brain mapping. Neuroimage, 21(1), 99-111. doi: 10.1016/j.neuroimage.2003.08.026

[5]. Hoshi, Y., Kobayashi, N., Tamura, M. (2001). Interpretation of near-infrared spectroscopy signals: a study with a newly developed perfused rat brain model. Journal of Applied Physiology, 90(5), 16571662.

[6]. Strangman, G., Culver, J. P., Thompson, J. H., Boas, D. A. (2002). A Quantitative Comparison of Simultaneous BOLD fMRI and NIRS Recordings during Functional Brain Activation. Neuroimage, 17(2), 719-731. doi: 10.1006/nimg.2002.1227 